\title{
Determinants of compliance with iron supplementation among pregnant women in Senegal
}

\author{
Binetou C Seck* and Robert T Jackson \\ University of Maryland, Department of Nutrition and Food Science, 0112 Skinner Building, College Park, \\ MD 20742, USA
}

Submitted 15 March 2007: Accepted 9 July 2007: First published online 3 September 2007

\begin{abstract}
Background: Community iron supplementation programmes for pregnant women have lacked effectiveness, partly because of low compliance.

Objective: To determine factors that influence compliance among pregnant women in Senegal.

Design: Two hundred and twenty-one pregnant women, recruited from six health centres in Dakar during their first prenatal visit, were randomly assigned to receive either a prescription to purchase iron/folic acid tablets (control, $n=112$ ) to be taken daily, according to official policy, or to receive free tablets (treatment, $n=109$ ). Compliance was assessed 20 weeks after enrolment through interviews and pill count. Women with low or high compliance $(<70 \%$ or $\geq 70 \%)$ were asked to explain what influenced their adherence to supplementation.

Results: Overall compliance was 69\%; it was significantly higher in the treatment than in the control group ( $86 \%$ vs. $48 \%$; $P<0.0001)$. Women with high compliance (58\%) were motivated by: (1) the perception of improved health upon taking the tablets (treatment $=24 \%$, control $=10 \%$ ); (2) the insistence by midwives that they take the tablets; and (3) the mention that the tablets would improve health. Women with low compliance (42\%) reported: (1) the experience of side-effects that they associated with the tablets (treatment $=13 \%$, control $=$ 14\%); (2) misunderstanding that they needed to continue taking the tablets throughout pregnancy (treatment $=0 \%$, control $=18 \%$ ); and (3) forgetfulness. Conclusion: Compliance with iron/folic acid supplementation in Senegal can be increased by providing women with clear instructions about tablet intake and educating them on the health benefits of the tablets.
\end{abstract}

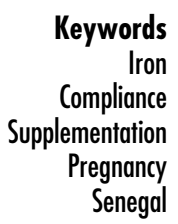

The World Health Organization (WHO) estimates that anaemia affects $>47 \%$ of pregnant women in West Africa and that more than half of the cases are due to iron deficiency (ID) ${ }^{1,2}$. In Senegal the prevalence of anaemia is even higher $(>50 \%)^{3-5}$. Anaemia has adverse consequences including fatigue, decreased work capacity, and poor pregnancy outcomes such as preterm birth, low birth weight, and increased risk of maternal death during both delivery and the postpartum period ${ }^{6-8}$.

To combat ID and anaemia, many West African countries, including Senegal, have implemented iron supplementation programmes for pregnant women? ${ }^{\text {. However, }}$ although clinical trials have repeatedly shown the efficacy of supplementation ${ }^{10}$, most if not all community-based programmes have not been shown to be effective, i.e. they have not decreased the incidence and prevalence of anaemia when deployed in the field. Studies conducted in South-East Asia, Latin America and in only a few African countries have shown that one of the main reasons why these programmes have been less effective than anticipated is low compliance of women with taking daily iron supplements. Low compliance has been associated with a number of factors, including: (1) gastrointestinal sideeffects that can occur with taking iron; (2) inadequate supply of tablets (including limited resources to purchase tablets); (3) inadequate counselling of patients by healthcare providers concerning the utility of tablets and possible transient side-effects; (4) poor utilisation of prenatal health-care services; (5) lack of knowledge and/or patient fears about the tablets; and (6) community beliefs, attitudes and practices that affect women's perception regarding tablet use $\mathrm{e}^{11-14}$.

In Senegal, all women who seek prenatal care at government-run public health centres are given a written prescription by midwives to purchase iron/folic acid tablets that contain $65 \mathrm{mg}$ of elemental iron $(200 \mathrm{mg}$ ferrous sulphate) and $250 \mu \mathrm{g}$ of folic acid. The tablets are sold at the health centre pharmacies and cost about 1 US 
cent per 10 tablets. The supplementation programme in Senegal has been in place for nearly two decades; however, the overall prevalence of anaemia among pregnant women who attend prenatal centres has not declined ${ }^{4}$.

The present study is part of a larger study which sought to compare the effectiveness of the current Senegalese programme with the effectiveness of a modified supplementation strategy whereby women would receive a free supply of iron/folic acid tablets from the hands of the midwives during the prenatal visit instead of a prescription to purchase the tablets from the pharmacist. The hypothesis was developed that women receiving free tablets would eliminate several potential barriers to programme success, thereby increasing compliance. In the randomised controlled trial we found that women who received free tablets did have a significantly higher compliance $(76 \%)$ than women who received prescriptions (61\%) (BC Seck and RT Jackson, Improving the effectiveness of the iron supplementation program for pregnant women in Senegal: a randomized trial, unpublished).

The purpose of the present study was to determine and understand the specific factors that influence compliance with supplementation among pregnant women in Senegal; such understanding is crucial to the design and implementation of a more successful and effective programme to prevent and treat anaemia.

\section{Subjects and methods}

\section{Study area and population}

The study was conducted in Dakar, the capital of Senegal, home to $22 \%$ and $80 \%$ of the total and urban populations, respectively. Senegalese women have a high fecundity rate of 6 children, which accounts in part for a population rate of growth of $3 \%$. Low birth weight is prevalent (15\%), and infant and maternal mortality rates are high (58/1000 and 560/100000, respectively). Ninety-eight per cent of women aged 20-34 years in Dakar seek antenatal care, the vast majority from public health centres ${ }^{15}$. This care is provided by licensed midwives who receive a three-year training course at government-accredited midwifery schools. Prenatal care is highly subsidised by the Government and thereby made affordable so that virtually everyone has access to it: the first visit costs $\$ 2.7$ on average and subsequent visits cost $\$ 1$ each.

Dakar is subdivided into eight health districts, which have a total of 11 health centres. Each district represents a different geographical area ${ }^{16}$. We chose the 'reference' health centres in five health districts: two of the selected health centres were located in the South health district; the remaining four were each located in the Centre, West, Pikine and Guediawaye health districts. Each health centre covers a population of about 180000 individuals, the vast majority of whom are residents of Dakar ${ }^{16}$. The remaining three health districts do not have 'reference' health centres and therefore were not chosen. 'Reference' health centres are larger than other health centres, are ethnically and economically representative of the lowincome population of Dakar, are equipped to provide more sophisticated medical care such as radiography and surgery, and they train future health-care workers. The randomisation procedures used in the larger study are described in detail elsewhere (BC Seck and RT Jackson, Improving the effectiveness of the iron supplementation program for pregnant women in Senegal: a randomized trial, unpublished). Briefly, the health centres were randomly assigned using a computerised random digit generator to either: (1) dispense the routine prenatal visit services during which a prescription to purchase iron/ folic acid tablets ( $65 \mathrm{mg}$ elemental iron $+250 \mu \mathrm{g}$ folic acid) was given to women (control group; $n=3$ centres), with instructions to purchase as many tablets as they could afford after their first visit, to ingest one tablet per day, and to refill the prescription as necessary until their follow-up visit; or (2) dispense the routine prenatal visit during which the midwife gave women a free supply of tablets with instructions to take one tablet per day until the end of pregnancy (treatment group; $n=3$ centres). The tablets were purchased from the National Pharmacy, which supplies the health centre pharmacies with tablets of the same appearance and composition as are for sale exclusively at the health centre pharmacies. The researchers pre-packaged the tablets in plastic bags which contained 200 tablets each (sufficient for the duration of pregnancy), that were given to the midwives prior to the beginning of data collection. Women in the treatment group were asked to keep empty tablet packages in the plastic bag that was provided. Study participants in both groups were instructed by the midwives to take the tablets with food or at mealtime. At the conclusion of data collection, all women who were seen at follow-up were given free tablets for daily supplementation for at least 3 months postpartum.

All women who registered for prenatal care in the six study centres, who had not used iron supplements prior to enrolment, who were 12 to 16 weeks' pregnant (as determined by fundal height and date of last menstrual period), and who were apparently healthy, were invited to participate in the study after giving informed consent. Women, at baseline, who had severe anaemia (haemoglobin $(\mathrm{Hb})<70 \mathrm{gl}^{-1}$ ) were referred for treatment and were excluded from the study.

This study was reviewed and approved by the Committee on Ethics of the Senegalese Ministry of Health and by the Institutional Review Board of the University of Maryland.

\section{Baseline characteristics}

Demographic data (age, ethnic background, education) were gathered using structured questionnaires. Medical 
and obstetric histories were obtained through interviews and by consulting patient health centre records when available. For face validity, all questionnaires that were used were pre-tested in 10 women from each health centre and all necessary changes were made prior to the start of actual data collection.

\section{Compliance}

Compliance was assessed 20 weeks after the date of enrolment. Women were contacted by telephone and given appointments to return to the health centre where they had originally registered for prenatal care. This means of communication was chosen because most women could not provide an exact street address, as is common in low-income areas of Dakar. They were asked to provide a phone number where they could be reached; it could be their home or mobile phone number, their husband's mobile phone number or a neighbour's or a relative's mobile/home phone number. All study participants, except for 13 women, were able to provide a phone number at enrolment (97\% of total sample). On average, three attempts were made to contact the participants; once they were reached, they were given an appointment date and time to return to the health centre for follow-up. For women who did not come to their first appointment, two subsequent attempts were made to schedule another appointment if needed.

During the follow-up visit, women in the control group were interviewed to determine the number of iron/folic acid tablets they had purchased since their first prenatal visit and the number of tablets that remained in their supply. In addition, women were asked whether they had actually ingested the missing tablets to ensure that the tablets had not been given away to friends or relatives or lost. Compliance in this group was then calculated as: (Number of tablets ingested/Number of tablets prescribed) $\times 100$, with the 'Number of tablets prescribed' determined from: (Date of follow-up minus Date of enrolment), where it was assumed that the number of days that had elapsed between enrolment and follow-up corresponded to the number of tablets that the patient should have ingested.

Women in the treatment group were asked to bring the bag of supplements they were given at enrolment to their follow-up visit, including the empty tablet packages. The number of tablets left in the bag was determined and women were questioned about actual tablet ingestion. Compliance was calculated as: (Number of tablets ingested/Number of days elapsed between enrolment and follow-up) $\times 100$. During the follow-up visit, each subject's compliance was computed and classified as either 'low compliance' (i.e. $<70 \%$ of tablets ingested) or 'high compliance' (i.e. $\geq 70 \%$ of tablets ingested). This classification was chosen on the basis of the WHO recommendation stating that, in countries where the prevalence of anaemia among pregnant women is $\geq 40 \%$, supplementation should be given for 6 months (24 weeks) of pregnancy and for 3 months postpartum, i.e. 168 tablets $^{17}$. For this study, participants were monitored over a period of 20 weeks during which the maximum number of tablets they should have ingested as directed would have been 140; $70 \%$ of 140 is 98 tablets, which is $83 \%$ of the WHO recommended number. The remaining 28 tablets that women would have consumed if monitoring was continued over the full 24 weeks would account for about 100\% compliance.

A semi-structured interview, which lasted $20 \mathrm{~min}$ on average, was then conducted with each subject. Women with low compliance were asked to identify the reason or reasons why they did not take the iron/folic acid tablets as directed most of the time. Women with high compliance were asked to identify the factor or factors that motivated them to take the tablets as directed most of the time. All interviews were conducted in the major local language of Senegal, Wolof. The questions were open-ended and were asked in a non-threatening or non-accusatory manner. Women's answers were recorded and then transcribed word for word by the interviewer. For example, women in the study population often refer to anaemia as 'lacking blood'; if a woman answered 'I took the tablets because I thought they would increase my blood', the interviewer wrote down the exact phrase. The translation and interpretation was done during data entry and analysis. Where women identified more than one factor that influenced their compliance, all factors were recorded; however, we noted the factor they identified as the strongest determining factor.

\section{Analysis of blood samples}

Venous blood samples were collected at enrolment and then at follow-up, 20 weeks later. $\mathrm{Hb}$ concentration was measured with the HemoCue photometer. Anaemia was defined as $\mathrm{Hb}<105 \mathrm{gl}^{-1}$ and $<110 \mathrm{gl}^{-1}$ in the second and third trimester of gestation, respectively ${ }^{18}$.

Erythrocyte protoporphyrin (EP) was measured at enrolment and follow-up with a portable front-face haematofluorometer (Aviv Biomedical). EP $>70 \mu \mathrm{mol} \mathrm{mol}^{-1}$ haem was considered abnormal ${ }^{19}$. Venous blood was also collected for analysis of serum ferritin (SF) at enrolment and follow-up. Serum samples were then obtained and analysed for SF concentration by enzyme-linked immunoassay with monoclonal antibodies using a kit and the Mini-Vidas (Biomerieux). An SF concentration $<15 \mu \mathrm{gl}^{-1}$ was considered abnormal ${ }^{20}$. ID was defined as

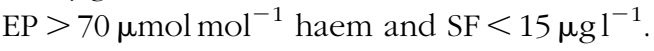

\section{Statistical analysis}

The questionnaires were coded and the data entered into SPSS (version 15.0; SPSS Inc.). Each compliance 
questionnaire was analysed to determine the key reason why the subject had a low or high compliance and that reason was assigned an individual code; the frequency distribution of the different reasons was then calculated. To correct data entry errors, frequency analyses for all the variables were checked as well as variable ranges. Student $t$-tests were used to compare means; $\chi^{2}$ tests of homogeneity were used to compare compliance between women who received prescriptions (control) and women who received free tablets (treatment) and the proportions of anaemia and ID between the two groups. To assess the significance of change in the proportion of women with anaemia and women with ID from baseline to follow-up within the two groups, the McNemar test was performed. Statistical significance was set at $P<0.05$ for all analyses.

\section{Results}

\section{Participation}

Of the 366 women who participated in the study, 221 (60\%) had complete information on compliance and were included in the analysis. There were no significant differences in background data (socio-economic status, parity, gravidity, etc.) between women who were included and those who were not (data not shown). Figure 1 shows the number of subjects recruited in each centre.

\section{Sociodemographic, bealth and baematological characteristics}

The mean age of the sample was $27 \pm 6$ years. Most of the women were married (94\%) and 57\% were illiterate. Seventy-six per cent reported never having been diagnosed with a major illness such as HIV/AIDS, cancer or diabetes (the rest of the sample reported illnesses such as asthma, chronic heartburn and migraines); 51\% described their state of health at the time of enrolment as either 'good' or 'excellent'. The control and treatment groups did not differ by age, parity, gravidity, stage of gestation, weight or height at baseline. The women had achieved, on average, $4.4 \pm 4.2$ years of formal education and $71 \%$ were not employed at the time of enrolment (Table 1). Table 2 shows the sample's ethnic distribution.

\section{Overall compliance and determinants}

The overall compliance in the control and treatment groups was $69 \pm 29 \%(n=221) ; 42 \%$ of the women had 'low' compliance (<70\%: mean $42 \pm 21 \%, n=93$ ) and 58\% had 'high' compliance $(\geq 70 \%$ : mean $88 \pm 13 \%$, $n=128$ ). Among the compliant women, the four main reasons reported for taking the tablets as directed most of the time were: (1) that they perceived a health benefit when they took the tablets and therefore continued taking them (i.e. alleviation of fatigue and dizziness and improved appetite; $32 \%$ of answers); (2) because the midwife directing them to take the tablets was a strong motivator to do so (19\% of answers); (3) because the midwife specifically indicated that the tablets would improve their health (13\% of answers); and (4) that they always take prescribed medications because they are fearful of illness (11\% of answers). About 14\% of the women stated that they knew that the tablets would 'increase their blood' (remedy anaemia) or prevent them from 'losing blood' (prevent anaemia), which is what motivated compliance (Table 3).

Among women who had low compliance $(<70 \%)$, the five main reasons why they did not take the tablets as directed most of the time were: (1) that they experienced gastrointestinal side-effects (i.e. one or a combination of nausea, vomiting, diarrhoea and constipation; $27 \%$ of answers); (2) that the midwife did not explain or they did not understand that they needed to purchase more tablets after their first prescription ran out (18\% of answers; this answer is relevant only for the control group); (3) that they forgot to take the tablets most days (17\% of answers);

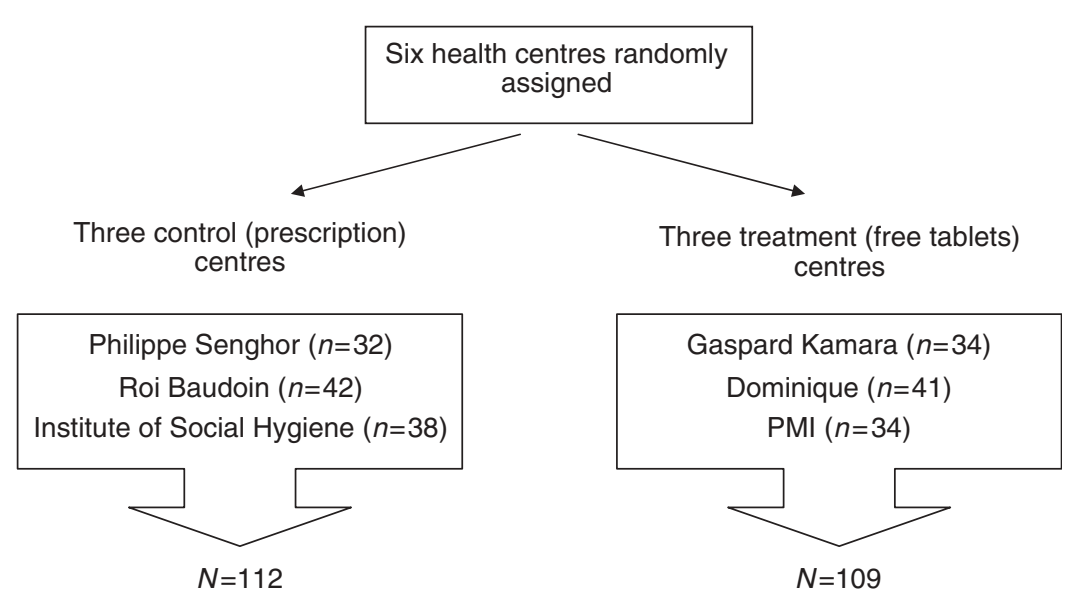

Fig. 1 Number of women in the control and treatment groups with complete haematological and compliance data 
Table 1 Baseline characteristics of women in the treatment and control groups

\begin{tabular}{lcccc}
\hline Variable & All $(n=221)$ & Control group $(n=122)$ & Treatment group $(n=109)$ & $P$-value \\
\hline Age (years) & $26.7 \pm 0.4$ & $26.6 \pm 0.6$ & $27.3 \pm 0.6$ & $0.7 \pm 0.4$ \\
No. of years of formal education & $4.4 \pm 0.3$ & $4.2 \pm 0.4$ & 26.6 & 0.350 \\
\% Employed & 29.4 & 32.1 & $0.99 \pm 0.07$ & $0.366+$ \\
Amount spent on food daily (\$) & $0.97 \pm 0.05$ & $0.95 \pm 0.82$ & $2.9 \pm 0.2$ & 0.663 \\
Gravidity & $3.0 \pm 0.1$ & $3.2 \pm 0.2$ & $1.6 \pm 0.2$ & 0.305 \\
Parity & $1.6 \pm 0.1$ & $1.8 \pm 0.2$ & $11.8 \pm 0.4$ & 0.458 \\
Stage of gestation (weeks) & $11.8 \pm 0.3$ & $11.8 \pm 0.4$ & $61.4 \pm 1.3$ & 0.930 \\
Weight (kg) & $61.0 \pm 0.9$ & $60.4 \pm 1.2$ & $1.65 \pm 0.003$ & 0.560 \\
Height $(\mathrm{m})$ & $1.64 \pm 0.003$ & $1.64 \pm 0.003$ & & 0.158 \\
\hline
\end{tabular}

Values are mean \pm standard error of the mean, except for $\%$ employed.

*Student's $t$-test $P$-value of the mean difference between treatment and control groups.

tPearson $\chi^{2}$ test of significance.

Table 2 Ethnic distribution of the sample of pregnant women in Dakar, Senegal $(n=221)$

\begin{tabular}{lcr}
\hline Ethnic group & Frequency & $\%$ \\
\hline Wolof/Lébou & 89 & 40.3 \\
Poular & 56 & 25.3 \\
Sérère & 34 & 15.4 \\
Dioula & 11 & 5.0 \\
Other & 31 & 14.0 \\
\hline
\end{tabular}

(4) that they could not afford to purchase the tablets (13\% of answers; this answer is relevant only for the control group); and (5) that they were tired of having to take so many tablets (11\% of answers). In $4 \%$ of the cases, the midwife should have, but did not, prescribe the tablets (Table 4).

\section{Compliance and determinants by group assignment}

Women who received free iron/folic acid tablets (treatment) ingested more than twice as many tablets $(P<0.0001)$ than were reported by women who received a prescription (control). This corresponded to a compliance of $86 \%$ in the treatment group and $48 \%$ in the control group, a difference that was very highly statistically significant $(P<0.0001)$.

\section{Control group}

Of the 112 women in the control group, $42 \%$ had compliance $\geq 70 \%$ (mean $88 \pm 10.5 \%, n=54$ ). The four main reasons why women took the tablets as directed most of the time were: (1) the fact that the midwife directed them to do so (24\% of answers); (2) that they always take prescribed medications because they are fearful of illness (20\% of answers); (3) that they perceived a health benefit when they took the tablets and therefore continued taking them (18\% of answers); and (4) the fact that the midwife specifically indicated that the tablets would improve their health (17\% of answers). About 10\% of the women knew that the tablets would 'increase their blood' (remedy anaemia) or prevent them from 'losing blood' (prevent anaemia), which is what motivated compliance (Table 3).

Fifty-eight per cent of women in the control group had compliance $<70 \%$ (mean $36 \pm 21 \%, n=58$ ). The four main reasons why they did not take the tablets as directed most of the time were: (1) that the midwife did not explain or they did not understand that they needed to purchase more tablets after their first prescription ran out (28\% of answers); (2) that they experienced gastrointestinal side-effects (22\% of answers); (3) that they could not afford to purchase their prescription $(21 \%$ of answers); and (4) that they forgot to take the tablets most days (12\% of answers). In 3\% of the cases, the midwife should have, but did not, prescribe the tablets (Table 4).

\section{Treatment group}

Of the 109 women in the treatment group, 68\% had compliance $\geq 70 \%$ (mean $88 \pm 15 \%, n=74$ ). The three main reasons why women took the tablets as directed most of the time were: (1) that they perceived a health benefit when they took the tablets and therefore continued taking them ( $42 \%$ of answers); (2) the fact that the midwife directed them to do so (15\% of answers); and (3) the fact that the midwife specifically indicated that the tablets would improve their health (11\% of answers). About $18 \%$ of the women knew that the tablets would 'increase their blood' (remedy anaemia) or prevent them from 'losing blood' (prevent anaemia), which is what motivated compliance; $8 \%$ of the women felt that the tablets had to be important since they were given to them during the prenatal visit (Table 3).

Thirty-two per cent of women in the treatment group had compliance $<70 \%$ (mean $51 \pm 19 \%, n=35$ ). The three main reasons why they did not take the tablets as directed most of the time were: (1) that they experienced gastrointestinal side-effects (34\% of answers); (2) that they forgot to take the tablets most days (26\% of answers); and (3) that they grew tired of taking the tablets (23\% of 
Table 3 Factors that influenced compliance with supplementation with iron/folic acid among pregnant women with high compliance ( $\geq 70 \%)$ in the overall sample and in the control and treatment groups

\begin{tabular}{|c|c|c|c|c|c|c|}
\hline \multirow[b]{2}{*}{ Motivating factor } & \multicolumn{2}{|c|}{ Overall sample $(n=128)^{\star}$} & \multicolumn{2}{|c|}{ Control group $(n=54) \dagger$} & \multicolumn{2}{|c|}{ Treatment group $(n=74) \ddagger$} \\
\hline & Frequency & $\%$ & Frequency & $\%$ & Frequency & $\%$ \\
\hline Felt better when they ingested the tablets & 41 & 32.0 & 10 & 18.5 & 31 & 41.8 \\
\hline Midwife instructed them to take the tablets & 24 & 18.8 & 13 & 24.0 & 11 & 14.8 \\
\hline Midwife explained that tablets would improve health & 17 & 13.3 & 9 & 16.6 & 8 & 10.8 \\
\hline Always take whatever they are prescribed because of fear of illness & 14 & 10.9 & 11 & 20.4 & 3 & 4.0 \\
\hline Knew that the tablets would 'increase their blood' & 8 & 6.2 & 2 & 3.7 & 6 & 8.1 \\
\hline Were afraid of 'losing blood' & 6 & 4.7 & 1 & 1.9 & 5 & 6.8 \\
\hline Since the tablets were given to them, they felt it was important to take them & 6 & 4.7 & - & - & 6 & 8.1 \\
\hline Husband/mother felt tablets were very important for their health and made sure they took them & 5 & 3.9 & 3 & 5.6 & 2 & 2.6 \\
\hline Knew that they 'lacked blood' and that the tablets would increase it & 4 & 3.2 & 2 & 3.7 & 2 & 2.6 \\
\hline Thought that the tablets would make them feel better & 3 & 2.2 & 3 & 5.6 & - & - \\
\hline
\end{tabular}

${ }^{*}$ Total number of women in overall sample $=221$ (total $\%$ may not equal 100 due to rounding)

†Total number of subjects in control group $=112$.

$\neq$ Total number of subjects in treatment group $=109$

Table 4 Factors that influenced compliance with supplementation with iron/folic acid among pregnant woment with low compliance ( $<70 \%)$ in the overall sample and in the control and treatment froups

\begin{tabular}{|c|c|c|c|c|c|c|}
\hline \multirow[b]{2}{*}{ Motivating factor } & \multicolumn{2}{|c|}{ Overall sample $(n=93)^{*}$} & \multicolumn{2}{|c|}{ Control group $(n=58)+$} & \multicolumn{2}{|c|}{ Treatment group $(n=35) \ddagger$} \\
\hline & Frequency & $\%$ & Frequency & $\%$ & Frequency & $\%$ \\
\hline Experienced gastrointestinal side-effects & 25 & 26.8 & 13 & 22.5 & 12 & 34.2 \\
\hline $\begin{array}{l}\text { Took the tablets until the prescription ran out, but did not } \\
\text { purchase more tablets because the midwife did not say so }\end{array}$ & 17 & 18.3 & 17 & 29.3 & - & - \\
\hline Forgot to take the tablets on most days & 16 & 17.2 & 6 & 10.3 & 9 & 25.7 \\
\hline Could not afford to purchase the prescription & 12 & 12.9 & 12 & 20.6 & - & - \\
\hline Grew tired of taking tablets & 11 & 11.8 & 3 & 5.1 & 8 & 22.8 \\
\hline Midwife did not prescribe/give the tablets (patient is correct) & 4 & 4.3 & 2 & 3.2 & 2 & 5.7 \\
\hline Lost the prescription/tablets and were afraid to ask for another & 3 & 3.2 & 2 & 3.2 & 2 & 5.7 \\
\hline Midwife did not prescribe the tablets (patient is mistaken) & 2 & 2.1 & 2 & 3.2 & - & - \\
\hline Was afraid that too many tables would harm her and/or her baby & 1 & 1.1 & - & - & 1 & 2.8 \\
\hline $\begin{array}{l}\text { Never came to follow-up prenatal visits and therefore could not } \\
\text { get another prescription to purchase tablets }\end{array}$ & 1 & 1.1 & 1 & 1.7 & - & - \\
\hline Gave away many tablets & 1 & 1.1 & - & - & 1 & 2.8 \\
\hline
\end{tabular}

${ }^{*}$ Total number of women in overall sample $=221$ (total \% may not equal 100 due to rounding)

tTol number of subjects in treatment group $=109$. 
Table 5 Prevalence of anaemia and iron deficiency at baseline and follow-up in women who received a prescription (control) and women who received free iron tablets (treatment)

\begin{tabular}{lcc}
\hline & $\begin{array}{c}\text { Control group } \\
(n=112)\end{array}$ & $\begin{array}{c}\text { Treatment group } \\
(n=109)\end{array}$ \\
\hline $\begin{array}{l}\text { \% Anaemic at baseline* } \\
\% \text { Anaemic after 20 }\end{array}$ & 37.0 & 25.6 \\
$\quad$ weekst & $62.0 \S$ & $31.0 \|$ \\
$\begin{array}{l}\text { \% Iron-deficient at } \\
\text { baselinef }\end{array}$ & 28.3 & 28.9 \\
$\begin{array}{l}\text { \% Iron-deficient after 20 } \\
\text { weeks }\end{array}$ & 49.0 ฯ & $21.1^{\star *}$ \\
\end{tabular}

*Haemoglobin $(\mathrm{Hb})<105 \mathrm{gl}^{-1}$.

$+\mathrm{Hb}<110 \mathrm{gl}^{-1}$.

¥Erythrocyte protoporphyrin $>70 \mu \mathrm{mol} \mathrm{mol}^{-1}$ haem and serum ferritin $<15 \mu \mathrm{gl}^{-1}$.

$\S$ McNemar test $(P<0.001)$; significantly different from treatment, $\chi^{2}$ test $(P<0.0001)$

$\checkmark$ McNemar test $(P=0.001)$; significantly different from treatment, $\chi^{2}$ test $(P<0.001)$.

$\|$ McNemar test $(P=0.442)$.

${ }^{*}$ McNemar test $(P=0.248)$.

answers). In 19\% of cases either the midwife did not give the free tablets to the women or the women themselves lost them or gave them away to friends and/or relatives (Table 4).

\section{Prevalence of anaemia and ID in the control and treatment groups at follow-up}

Twenty weeks after enrolment, the proportion of women with anaemia increased significantly among women who received a prescription (control) (37\% vs. 62\%; $P<0.001)$, but did not increase significantly among women who received free tablets (treatment) $(26 \%$ vs. $31 \% ; P=0.442)$. The proportion of women who had ID increased significantly in the control group (28\% vs. $49 \% ; P=0.001$ ) but decreased in the treatment group (29\% vs. 21\%; $P=0.248)$. The prevalence of anaemia and ID was significantly higher among women who received prescriptions than in those who received free tablets (Table 5). Mean differences in $\mathrm{Hb}$ and $\mathrm{EP}$ concentrations remained significant after controlling for baseline $\mathrm{Hb}, \mathrm{EP}$ and SF, and baseline and follow-up stage of gestation, in a multivariate analysis of covariance.

\section{Discussion}

This study is the first attempt to understand the factors that influence adherence to iron supplementation among pregnant women in Senegal. It is also one of the few studies that examine the specific factors that facilitate high compliance with supplementation in a community environment anywhere in the world. The study sample was drawn from six different public health centres that each cater to a large group of low-income women residing in different neighbourhoods of Dakar. The ethnic and socio-economic distribution of the sample is similar to that of the general population of Senegal ${ }^{15}$. The sample size was large compared with other studies of this kind.

Compliance with supplementation in the entire sample was 69\%; however, women who received free iron/folic acid tablets during the prenatal visit had significantly higher compliance than women who received a prescription, i.e. $86 \%$ vs. $48 \%$. The overall compliance of $69 \%$ is relatively high compared with compliance previously reported for pregnant women in developing countries $^{12-14,21-24}$.

In the overall sample, women with the highest compliance $(\geq 70 \%)$ were motivated by four major factors which accounted for $75 \%$ of the responses: (1) the perception of improved health upon taking the tablets; (2) the admonishment from midwives to take the tablets; (3) the specific mention by midwives that the tablets would improve their health; and (4) the fear of becoming ill.

The first factor in the overall sample was also the single most important motivator among women who received free tablets during the prenatal visit ( $42 \%$ of answers) but was only the third motivating factor among women who received prescriptions (18\% of answers). This strongly suggests that dispensing the tablets during the visit increases the likelihood that women will start iron/folic acid treatment rapidly and will continue long enough to reap health benefits, which in turn may motivate them to continue the treatment. For women who are given written prescriptions and directed to purchase the tablets, some barriers may prevent or delay them from complying, one of which is financial: a significant proportion (21\%) of women who had the lowest compliance in the control group indicated that they could not afford to purchase their prescriptions. Furthermore, a significant proportion of women in the same group indicated that they did not purchase and take more tablets after their first prescription ran out because they did not know that they were supposed to do so. This problem could not have occurred among women who received free tablets since they were given a supply for the remainder of their pregnancy; thus, the risk of an interruption of treatment in this group was decreased.

The second and third factors in the overall sample suggest that the quality of counselling during the prenatal visit is a very important determinant of compliance. The combination of midwives telling women to take the tablets and of midwives specifically explaining to them that the tablets will improve health accounted for 32\% of responses. Conversely, we found that among women who had the lowest compliance, 18\% either misunderstood the directions given by the midwife or the midwife's directions were unclear. Inadequate counselling by health-care providers has been documented by Galloway et $a l^{12,13}$ as a major barrier to compliance in eight other developing countries and by Oluwatoyin in Nigeria ${ }^{11}$. It was the single most important reason for non-compliance 
with supplementation as directed (28\% of answers) among women who received only prescriptions; whereas among women who received free tablets but had low compliance, inadequate counselling was not a factor. Researchers in Mali also found a strong link between the quality of counselling by health-care providers and compliance. They reported that pregnant women complied with supplementation when they were provided with 'minimum, consistent, and easily understandable information and counselling, ${ }^{25}$. Results in the current study suggest that giving the tablets to women during the prenatal visit may encourage the midwives to more clearly explain their purpose; this is further supported by the fact that $18 \%$ of women who had the highest compliance in the treatment group indicated that they took the tablets as directed most of the time because they knew their specific function, i.e. they knew that the tablets would either prevent them from becoming anaemic or would treat their existent anaemia. This knowledge was apparent in only $10 \%$ of women who received a prescription and had the highest compliance. Adequate verbal counselling is especially important for women with little or no formal education. On average, women in this sample had received only 4 years of formal education and most could not read or write. In this study, it was assumed that midwives at the treatment and control centres dispensed the same overall quality of prenatal care since they all received the same training at government-accredited midwifery schools.

The fourth motivating factor in the overall sample, the fear of becoming ill, cannot be tied to specific knowledge about the function of the iron/folic acid tablets. Women who evoked it as a factor indicated that they generally take any medication they are prescribed. It accounted for $20 \%$ of answers among women with the highest compliance in the control group but was not a major determinant among women with the highest compliance in the treatment group. This may suggest that the specific knowledge of the role of the tablets is a stronger motivator than just the fear of illness.

In the overall sample, women with the lowest compliance were influenced by five factors, which accounted for $81 \%$ of responses. The single most important factor was the experience of gastrointestinal side-effects $(27 \%$ of responses). Side-effects have traditionally been considered the major obstacle to compliance, leading many to advocate weekly instead of daily supplementation; however, more recently, studies have shown that sideeffects have a limited influence on compliance (especially when it is unclear how women distinguish the symptoms associated with taking iron tablets and those associated with pregnancy $)^{26}$. Findings in this study support this view because despite the fact that more women cited gastrointestinal side-effects as a major reason for low compliance among those who received free tablets than among those who received prescriptions, women who received free tablets still had a significantly higher compliance. This may be a further suggestion that adequate counselling is a stronger determinant of whether or not women adhere to supplementation. Midwives should explain to women that iron tablets should be taken with food and that side-effects associated with taking the tablets are generally transient. Although verbal exchanges between the patients and midwives during the prenatal visit were not studied, women who received free tablets may have received more thorough counselling.

The second important barrier to compliance, inadequate counselling, was discussed above. The third determinant of low compliance was forgetfulness among women with the lowest compliance in the overall sample (17\%) and both in the control group (12\%) and the treatment group (26\%). This problem could be addressed through better counselling during the prenatal visit by suggesting to women strategies to remember to take their tablets; for example, placing the tablets in a spot that they see every day (e.g. breakfast or night table, kitchen counter, etc.).

The fourth barrier was related to the financial inability of women to purchase the tablets; this only concerned women who received prescriptions rather than free tablets (21\% of responses). In fact, the written prescription that women are given during the prenatal visit contains items other than iron/folic acid tablets. The content of the prescription will vary according to individual patient afflictions; women who did not purchase the tablets for financial reasons generally did not purchase any other medication on the prescription, even though the iron/folic acid tablets are affordable (1 US cent per 10 tablets). In addition, among women in the treatment group who had the highest compliance, few indicated that the fact that the tablets were provided free of charge was a facilitator of compliance. The results from this study suggest that the benefit of iron/folic acid tablets being given during the prenatal visit lies more in the fact that it encouraged midwives to explain the purpose of the tablets and less in the fact that the tablets were free.

The fifth barrier to compliance was expressed by women as being 'tired of taking pills'. This sentiment was even stronger in women in the treatment group than in women in the control group. Women who expressed this sentiment were further questioned about the reason for this 'pill fatigue', but they did not give further details. This 'fatigue' may also explain why more women in the treatment group gave away tablets to friends and relatives or lost the tablets. This suggests that daily supplementation is impractical for certain individuals who may benefit more from weekly or bi-weekly supplementation.

Tablet ingestion was not supervised because the intention was to examine the determinants of compliance under usual field conditions. Calculations of compliance were mostly based on the number of tablets that women reported taking in the control group and on counting the 
number of tablets remaining in the bag of tablets that was given to women in the treatment group. However, the finding that women in the latter group had a significantly higher compliance is supported by the fact that women in this group also had a significantly improved iron status and a lower incidence of anaemia compared with women in the other group.

\section{Conclusions and recommendations}

Compliance of pregnant women with iron/folic acid supplementation in Senegal can be greatly improved by increasing women's access to the supplements. In this study, access was facilitated by asking midwives to hand the tablets to women (free of charge) during the prenatal visit, an effort which led to another facilitator of compliance, providing women with specific and concise information on the health benefits that the tablets confer. This information should include a mention of possible transient side-effects of iron/folic acid tablets and strategies for women to remember to take the tablets on a daily basis. Although the fact that the tablets were free facilitated compliance, it was not found to be as important a facilitator as proper counselling. Thus, modifying the Senegalese supplementation programme to increase compliance may not involve large additional governmental costs. It may just entail a re-routing of the tablet delivery system whereby the tablets would be distributed by the very people who are directly involved with delivering care to pregnant women, the health centre midwives, rather than the health centre pharmacists. However, pharmacy workers should also be trained to give appropriate counselling to women regarding tablet use.

Since adequate counselling is an important determinant of compliance, future inquiries about the effectiveness of iron/folic acid supplementation in Senegal and other countries should focus on the nature of the interaction between midwives and patients during the prenatal visit and on midwives' training and knowledge about anaemia.

\section{Acknowledgement}

This study was funded in part by the Donald Leishear International Program Fund and the General Research Board (University of Maryland).

\section{References}

1 World Health Organization (WHO). The Prevalence of Anemia in Women: A Tabulation of Available Information, 2nd ed. WHO/MCH/MSM 92.2. Geneva: WHO, 1992.

2 United Nations Children's Fund/World Health Organization (WHO). Prevention and Control of Iron Deficiency Anemia in Women and Children. Geneva: WHO, 1999.
3 Canadian International Development Agency. MICAH Phase 1 Results: 1995-01 [online]. Available at http:// www.worldvision.ca/home/articles/International_Programs/ MicahReportEnglish.pdf. Accessed July 2004.

4 Ndiaye O, Mbaye A, Diouf L, Sow H, Sylla A, Kuakuvin N, Fall M. Risk factors of low birth weight newborn: influence of maternal age, parity, gestation, nutritional status, and maternal pathology. Dakar Medical 1998; 43: 188-90.

5 Seck B. Prevalence and determinants of anemia in pregnant women in Dakar, Senegal. Masters thesis, University of Maryland, College Park, MD, 2002.

6 Rasmussen KM. Is there a causal relationship between iron deficiency or iron-deficiency anemia and weight at birth, length of gestation and perinatal mortality? Journal of Nutrition 2001; 131(Suppl.): 590S-603S.

7 Cogswell ME, Parvanta I, Ickes L, Yip R, Brittenham GM. Iron supplementation during pregnancy, anemia, and birth weight: a randomized controlled trial. American Journal of Clinical Nutrition 2003; 78: 773-81.

8 Scholl TO. Iron status during pregnancy: setting the stage for mother and infant. American Journal of Clinical Nutrition 2005; 81(Suppl.): 1218S-22S.

9 Yip R. Iron supplementation: country level experiences and lessons learned. Journal of Nutrition 2002; 132(Suppl.): 859S-61S.

10 Sloan NL, Jordan E, Winikoff B. Effects of iron supplementation on maternal hematologic status in pregnancy. American Journal of Public Health 2002; 92: 288-93.

11 Oluwatoyin E. Community attitudes to pregnancy, anemia, iron and folate supplementation in urban and rural Lagos, south-western Nigeria. Midwifery 2000; 16: 89-95.

12 Galloway R, McGuire J. Determinants of compliance with iron supplementation; supplies, side effects, or psychology? Social Science \& Medicine 1994; 39: 381-90.

13 Galloway R, Dusch E, Elder L, Achadi E, Grajeda R, Hurtado $\mathrm{E}$, et al. Women's perceptions of iron deficiency and anemia prevention and control in eight developing countries. Social Science \& Medicine 2002; 55: 529-44.

14 Jackson RT, Jackson LC. Biological and behavioral contributors to anemia during pregnancy in Liberia, West Africa. Human Biology 1987; 59: 585-97.

15 Macro International Inc. Enquête Démographique et de Santé au Sénégal (EDS-VI). Demographic and Health Surveys. Calverton, MD: Macro International Inc., 2005.

16 Ministere de la Sante Publique et de l'Action Sociale. Plan National de Développement Sanitaire et Social du Senegal (1998-2007) [online], 2007. Available at http://www.sante. gouv.sn/politiquesanitaire.php. Accessed February 2007.

17 Stoltzfus R, Dreyfuss ML. Guidelines for the Use of Iron Supplementation to Prevent and Treat Iron Deficiency Anemia. Washington, DC: International Nutritional Anemia Consultative Group/International Life Sciences Institute, 2000.

18 Centers for Disease Control and Prevention. CDC criteria for anemia in children and childbearing aged women. Morbidity and Mortality Weekly Report 1989; 38: 400-4.

19 World Health Organization (WHO). Iron Deficiency Anaemia: Assessment, Prevention, and Control - A Guide for Program Managers. WHO/NHD/01.3. Geneva: WHO, 2001.

20 Flowers CH, Kuizon M, Beard JL, Skikne BS, Covell AM, Cook JD. A serum ferritin assay for prevalence studies of iron deficiency. American Journal of Hematology 1986; 23: 141-51.

21 Dairo MD, Lawovin TO. Demographic factors determining compliance to iron supplementation in pregnancy in Oyo State, Nigeria. Nigerian Journal of Medicine 2006; 15: $241-4$. 
22 Carre N, Eono P, Kouakou K, Duponchel JL, Marquis M, Zahui KH. Iron supplementation associated with malaria prevention among pregnant women in Abidjan. Revue d'Épidemiologie et de Santé Publique 2003; 51: 31-8.

23 Young MW, Lupafva E, Kapenda E, Bobrow EA. The effectiveness of weekly iron supplementation in pregnant women of rural northern Malawi. Tropical Doctor 2000; 30 : 84-8.

24 Ekstrom EC, Kavishe FP, Habitcht JP, Frongillo EA, Rasmussen KM, Hemed L. Adherence to iron supplementation during pregnancy in Tanzania: determinants and hematological consequences. American Journal of Clinical Nutrition 1996; 64: 368-74.

25 Aguayo VM, Kone D, Bamba SI, Diallo B, Sidibe Y, Traore $\mathrm{D}$, et al. Acceptability of multiple micronutrient supplements by pregnant and lactating women in Mali. Public Health Nutrition 2005; 8: 33-7.

26 Hyder SM, Persson LA, Chowdhury AMR, Ekstrom EC. Do side-effects reduce compliance to iron supplementation? A study of daily- and weekly-dose regimens in pregnancy. Journal of Health, Population, and Nutrition 2002; 20: 175-9. 